\title{
Failed Flatfoot Reconstruction: Reasons and Management
}

\author{
Thanut Valleenukul ${ }^{1}$, Pongpol Petchkum ${ }^{2}$
}

\begin{abstract}
This article will focus on the common failures and the treatment plans for complications after flatfoot deformity correction procedures including how to prevent the failures. The comprehensive history and the physical examination, including a thorough assessment of which procedure was used throughout the prior surgery, are essential to realize this complex problem. The appropriate surgical procedure should be adjusted to the symptoms as it may be related to the cause of the failures from the previous operation.

Keywords: Arthrodesis, Complication, Failure, Flatfoot deformity, Osteotomy, Overcorrection, Reconstruction, Recurrence, Undercorrection. Journal of Foot and Ankle Surgery (Asia Pacific) (2021): 10.5005/jp-journals-10040-1150
\end{abstract}

\section{BACKGROUND}

A flatfoot deformity is a condition that there is the loss of the medial longitudinal arch with the failure of restraints coming from the diversities of deformity and disability. Additionally, the structural failures leading to other deformities include valgus hindfoot, abducted midfoot, and varus forefoot. ${ }^{1}$ The most common associated condition causing the deformity is posterior tibial tendon dysfunction (PTTD). Insufficiency of the posterior tibial tendon (PTT) results in the medial longitudinal arch collapse and the failure of the spring ligament, causing talonavicular (TN) subluxation and midfoot abduction. The unopposed peroneus brevis function due to the insufficiency of PTT strength leads to valgus deformity of hindfoot through the subtalar eversion. The valgus deformity of the hindfoot moves the Achilles tendon axis laterally compared to the subtalar joint axis. The Achilles tendon becomes an evertor of the subtalar joint causing more valgus hindfoot. In the advanced stage, the deltoid ligament failure may cause valgus instability of the ankle, leading to early degenerative change. ${ }^{2,3}$

The goals of treatment in the flatfoot deformity are realignment, pain relief, motion preservation, and over/undercorrection avoidance. The underlying causes of deformity and structural failure have to be addressed properly during surgical treatment to achieve good results and fewer complications. The undetected pathologies and inappropriate reconstruction will cause complications including recurrence of deformity, overcorrection, and persistent pain. ${ }^{3}$

\section{Evaluation of the Failed Flatfoot Reconstruction}

The evaluation of the patient with failed flatfoot reconstruction requires the history taking and the physical examination in detail to identify the area of the residual pain or deformity and the possible causes of them. The general health status of the patient should be examined for any systemic disease that can affect flatfoot progressions, such as, rheumatoid arthritis, diabetes, or Charcot neuroarthropathy. ${ }^{4}$ The alignment of hindfoot, midfoot, and forefoot should be evaluated in the standing position to detect any malalignment and deformity during weight-bearing. The residual hindfoot valgus or overcorrected varus, residual midfoot abduction, forefoot varus, and heel cord tightness must be detected and recognized preoperatively or intraoperatively to address all problems. The area of pain and tenderness at any part of the foot and ankle should be identified to detect joint arthritis or tendinitis. The motion of the peritalar region, midfoot, and ankle

\footnotetext{
1,2Department of Orthopedic Surgery, Bhumibol Adulyadej Hospital, Bangkok, Thailand
}

Corresponding Author: Thanut Valleenukul, Department of Orthopedic Surgery, Bhumibol Adulyadej Hospital, Bangkok, Thailand, Phone: +66818744111, e-mail: thanut1@yahoo.com

How to cite this article: Valleenukul T, Petchkum P. Failed Flatfoot Reconstruction: Reasons and Management. J Foot Ankle Surg (Asia Pacific) 2021;8(2):66-71.

Source of support: Nil

Conflict of interest: None

should be examined for the condition of arthrosis, tarsal coalition, or instability. The operative report of previous surgery should be reviewed for the intraoperative findings and the procedures being done including any complications that may affect the revision surgery.

The weight-bearing plain radiographs of foot and ankle including hindfoot alignment view ${ }^{5}$ are required to evaluate the radiographic alignment of the foot and review any joint condition, such as, arthrosis or coalition. The prior radiographs are useful to identify residual deformities or reconstructive failures of previous surgery.

The computed tomography (CT) scan is helpful to identify the nonunion at the osteotomy or arthrodesis site and the coalition of the subtalar joint.

The subtalar joint alignment and the calcaneofibular impingement are better determined by the weight-bearing CT (WBCT) scans. This technology may have meaningful suggestions for the treatment in the case of failure after the flatfoot reconstruction. ${ }^{6,7}$

Magnetic resonance imaging (MRI) can be used to figure out the medial soft tissue condition including the PTT, spring ligament, deltoid ligament, or flexor digitorum longus (FDL) tendon transfer condition.

\section{Reasons for the Reconstruction Failure}

A flatfoot deformity is a complex condition involving multiple structural problems that lead to a deformity in each part of the foot and ankle. The conservative treatment can be used successfully in the early stage with mild symptoms and may decelerate the progression of the disease. The surgical treatment is contemplated 
when conservative treatment is unsuccessful or in severe cases. Johnson and Strom's classification has been used widely to guide the procedure needed in each stage of PTTD. ${ }^{8}$ The surgical correction usually begins at the hindfoot followed by midfoot and then forefoot, respectively.

The procedures to correct hindfoot valgus may be the medial displacement calcaneal osteotomy (MDCO) or the subtalar arthrodesis depending on the condition of the subtalar joint. Either the lateral column lengthening $(\mathrm{LCL})$ by calcaneal opening wedge osteotomy (Evans) or the calcaneocuboid distraction arthrodesis may be used to correct midfoot abduction depending on the condition of the calcaneocuboid joint. Finally, either the medial cuneiform dorsal opening wedge osteotomy (Cotton's) or the first tarsometatarsal (TMT) arthrodesis may be the procedures to correct forefoot varus depending on the condition of the first TMT joint. ${ }^{9}$ The limited arthrodesis can be performed at the particular degenerative joint in the case with evidence of joint arthritis. The soft tissue procedures commonly used for PTTD are the tenosynovectomy of the PTT or FDL transfer, spring ligament repair or reconstruction, deltoid ligament reconstruction, gastrocnemius recession, or Achilles tendon lengthening depending on the reasons for reconstruction failure.

The surgical treatment for the flatfoot deformity may be unsuccessful for several reasons. The authors will define the common reasons for the failure and present the treatment plans for the mentioned problems.

\section{Failed Repair of the PTT and Flexor Digitorum Longus Transfer}

Regardless of the type of repair that was performed for the degenerated PTT, continuing symptoms or recurrence is possible when the underlying osseous deformities are not addressed. The failure of isolated tendon transfer or tenodesis can be from the continuing muscle imbalance when the Achilles tendon pull remains lateral to the subtalar joint axis and continues to be a valgus deforming force on the hindfoot. ${ }^{10}$ Flexor digitorum longus tendon transfer may eventually be a failure without bony procedures that provide the continuing biomechanical protection ${ }^{11}$ (Fig. 1).

The PTT repair will be unsuccessful if the musculotendinous unit is unworkable. Preserving the PTT can result in persistent symptoms and residual dysfunction. The viability of the musculotendinous unit can be demonstrated by the elasticity of the tendon. Simple debridement with PTT repair may be insufficient if tendinosis is noticeable. This situation usually requires complete removal of the degenerated PTT and tendon transfer should be determined as an adjunctive operation.

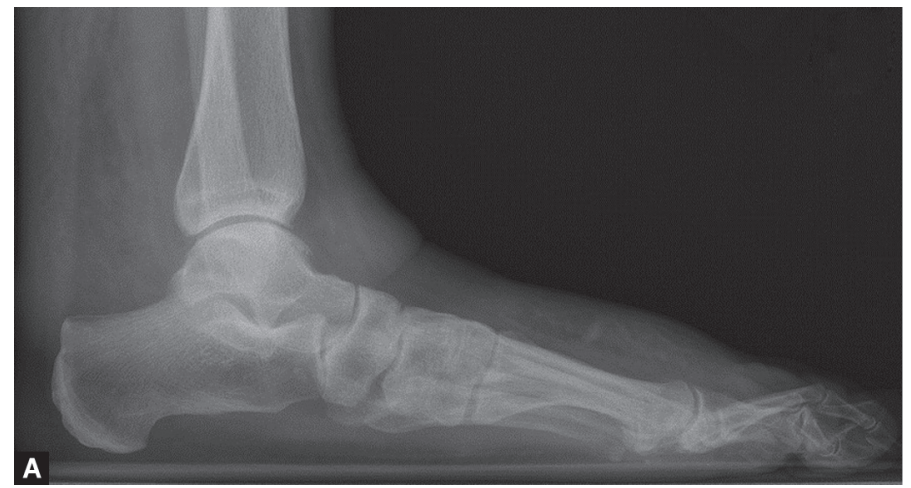

\section{Failure to Address the Equinus Component}

Almost all patients with the flatfoot deformity have some degrees of the equinus deformity. The failure to address the equinus deformity leads the surgeon to less than preferable results. ${ }^{12}$ The Achilles tightness can convert the triceps surae to the hindfoot evertor, causing additional stress on the medial structures and ensuing in more medial arch instability. The disregarding equinus component forces calcaneus into valgus position and restricts the subtalar joint inversion. The persistent equinus deformity can bring on substantial forces through the midfoot during axial loading with the consequent failure of the reconstruction. The gastrocnemius recession or Achilles lengthening can be performed in the same operation based on the intraoperative Silfverskiold test. ${ }^{13}$

\section{Medial Displacement Calcaneal Osteotomy Complications}

Failure in correction can be met with the joint preserving and the joint devastating procedures. The lack of success in deformity correction predisposes to the continuous pain, disability, and development of the flatfoot deformity. Determining all planes of deformity is crucial in the reconstructive procedures. The weightbearing radiographs are essential in the assessment of flatfoot deformity. The consideration of the talar head undercoverage by the navicular in AP view will indicate the amount of midfoot abduction.

Medial displacement calcaneal osteotomy can decrease strain on soft tissue in the medial aspect of the heel and alter the axis of action of the Achilles tendon. ${ }^{14}$ The medial translation of the calcaneal tuberosity for $8-10 \mathrm{~mm}$ is usually enough to correct hindfoot valgus and also restore the medial longitudinal arch. ${ }^{15}$ Medial displacement calcaneal osteotomy has been reported as an efficient surgical approach to manage mild to moderate cases of flatfoot deformity with stage II PTTD and negligible fixed forefoot supination. It may be inadequate to correct more severe cases of stage II PTTD.

The failure in correction from MDCO can arise from technical mistakes, particularly when the osteotomy is carried out too proximal. Displacement in this area can be impeded by the attachment of intrinsic muscles and plantar fascia in the tuber segment. The medial displacement can frequently be facilitated by the release of plantar structures.

The adequacy of MDCO with sufficient magnitude of the medial translation is required to prevent the failure. The medial longitudinal arch and midfoot abduction should be checked intraoperatively after MDCO for adequate correction. The failure

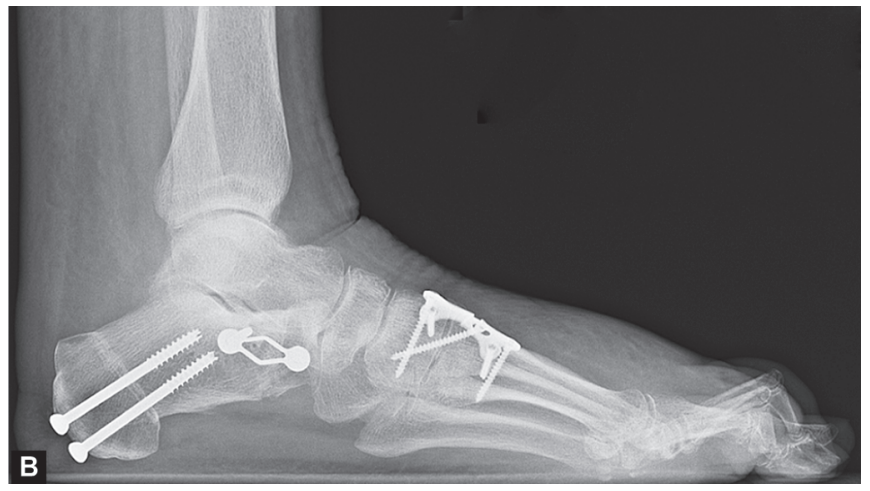

Figs 1 A and B: (A) A 54-year-old woman suffered from procedure failure after FDL tendon transfer to correct flexible flatfoot; (B) Revision surgery was accomplished with MDCO and LCL with Lapidus arthrodesis 
to correct the midfoot abduction leads to increased stress on the medial soft tissue causing the recurrence or failure of the flatfoot reconstruction. The $L C L$ should be considered if there is $>30 \% \mathrm{TN}$ uncoverage to improve the midfoot abduction and restore the medial arch. Lateral column lengthening is dispensable in the case with minimal midfoot abduction. ${ }^{16}$ However, many factors can result in the failure of the medial longitudinal arch restoration and midfoot abduction correction, such as, soft tissue condition or stiffness.

The overcorrection of MDCO changes the moment arm of the hindfoot alignment into the varus position. This situation may change the plantar pressure to the outer aspect of the foot which results in the symptoms from the excessive lateral load-bearing pressure or rigidity. It is crucial to evaluate the preoperative deformity magnitude and intraoperative soft tissue elasticity to obtain the proper result of the correction. The revision calcaneal osteotomy is suggested with caution for the risk of tibial nerve palsy. ${ }^{17}$

\section{Lateral Column Lengthening Complications}

The lateral column can be lengthened by the calcaneal opening wedge osteotomy (Evans) or the calcaneocuboid distraction arthrodesis. Both procedures entail the $L C L$ to improve the transverse plane deformity in the PTTD.

The undercorrection of Evans osteotomy can result from the inadequately intraoperative $L C L$ or the subsequent loss of correction from the autograft resorption. This condition can happen with the untimely weight-bearing situation, insufficient fixation, or incompatible graft composition. If the subsidence occurs, performing the revision lateral column procedures is acceptable. The locked plating fixation techniques to bridge the osteotomy is preferable to prevent further problems.

The overcorrection of Evans osteotomy may lead to the adducted midfoot position, the fifth metatarsal stress fracture, the traction neuritis, and the lateral foot pain. ${ }^{18}$ The lateral column overload can occur from the failure to recognize the forefoot varus deformity and the medial column instability. The study has also reported the increased pressure in the calcaneocuboid joint in the case of Evans osteotomy without definitive evidence for early joint degeneration in the long-term consequence. ${ }^{19}$ However, there are more frequent reports for the nonunion, the delayed union, the graft stress fractures, and the residual supination after arthrodesis of the lateral column. ${ }^{20,21}$ It is essential to ascertain whether there is the remaining forefoot supination before the revision surgery. The overcorrected lateral column may be restored either through the closing wedge osteotomy or the calcaneocuboid arthrodesis.

The nonunion can still occur after the $L C L$ within the calcaneus, although there is much less incidence of the nonunion in proportion to calcaneocuboid arthrodesis. (For more information on arthrodesis, see Arthithat Kirinpanu's article, "Role of arthrodesis in $A A F$ " in this issue). The nonunion can bring about the failure of correction, sagittal plane malalignment, and recurring flatfoot deformity. The management of nonunion or graft collapse frequently involves debridement, bone grafting, and fixation. Our preference treatment has been for the fresh frozen allograft together with the demineralized bone matrix (DBM). The graft has to be sizeable to reconstruct the alignment and compensate for the bone loss. The fixation is accomplished with the locking plate and screws.

\section{Failure to Determine the Subtalar or Subfibular Impingement}

The clinician can use WBCT scans to search for the subtalar impingement on the sagittal views or the subfibular impingement on the coronal views in the flatfoot deformity. The severe subtalar or subfibular impingement may necessitate the subtalar arthrodesis to place the foot in an appropriate position with the prevention of subsequent impingement or recurrence because the MDCO and LCL may not address these deformities.

\section{Residual Forefoot Supination}

The inability to address and reform the remaining forefoot varus or supination deformity after the hindfoot realignment may result in recurring flatfoot deformity and lateral column discomfort due to the lateral forefoot overload (Fig. 2). The forefoot supination occurs after chronic deformity in an attempt to compensate for the hindfoot valgus deformity and the medial arch collapse for maintaining the plantigrade position of the foot. ${ }^{22}$ If the residual forefoot supination is not addressed after the hindfoot reconstruction, this situation will force the midfoot into pronation as well as the hindfoot or ankle into a valgus position.

The evaluation of the forefoot supination should be done after the treatment of both hindfoot valgus and midfoot abduction because of the rigidity in conjunction with persistent supination of the forefoot. The residual forefoot supination results in the elevated pressure in the lateral aspect of the forefoot and the consequent overload. ${ }^{23}$ Either dorsal opening wedge osteotomy of medial cuneiform (Cotton's) or first TMT arthrodesis has been recommended to improve the residual forefoot deformity. The presence of first TMT joint instability or arthrosis will be beneficial to the surgical determination between the mentioned procedures.

\section{Failure to Address the Medial Column Instability}

The compensation of long-standing hindfoot valgus from the failure of PTT and the spring ligament complex throughout the medial column often happens at the first TMT joint, naviculocuneiform (NC) joint, or TN joint, leading to the loss of the medial column stability. The failure to recognize the instability of the medial column could result in ongoing deformity and inferior clinical consequences. The careful evaluation of the TN, NC, and TMT joints in the weight-

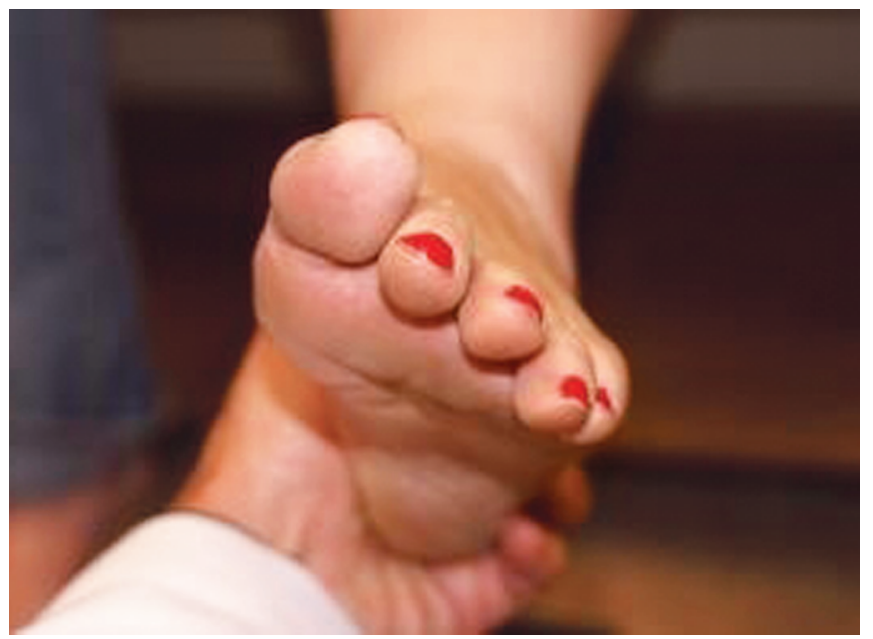

Fig. 2: Residual forefoot supination deformity was addressed following hindfoot reconstruction 

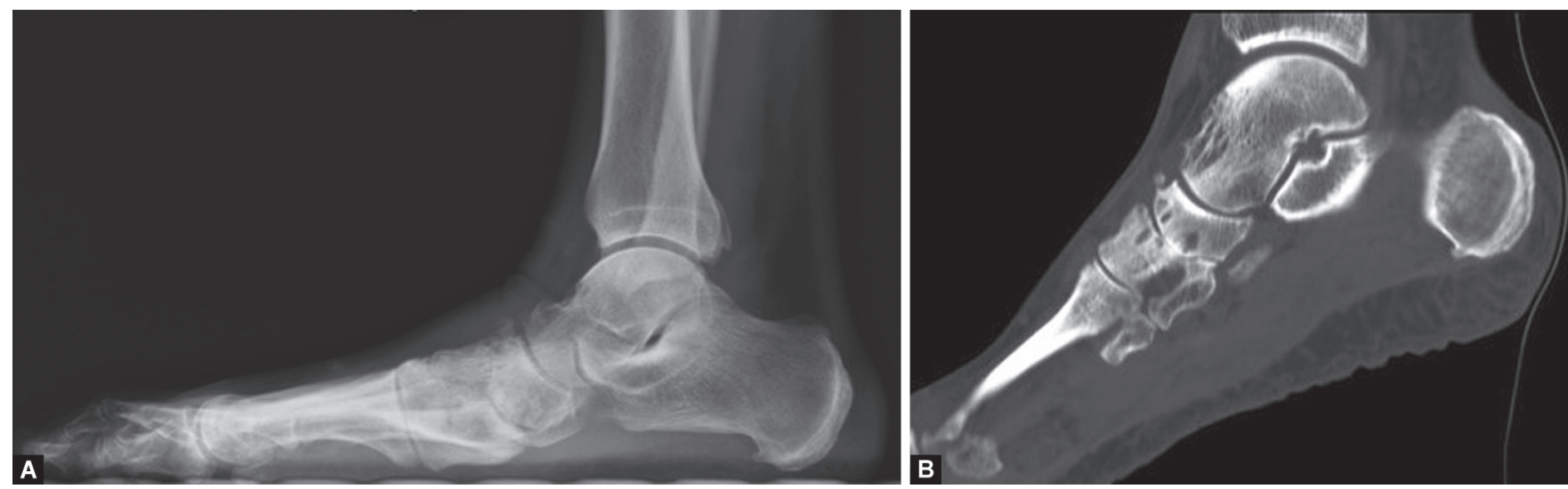

Figs $3 A$ and $B$ : (A) Lateral weight-bearing radiograph of 60-year-old man revealed naviculocuneiform sagging with dorsal joint space narrowing: (B) CT showed the degenerative changes at the naviculocuneiform joint

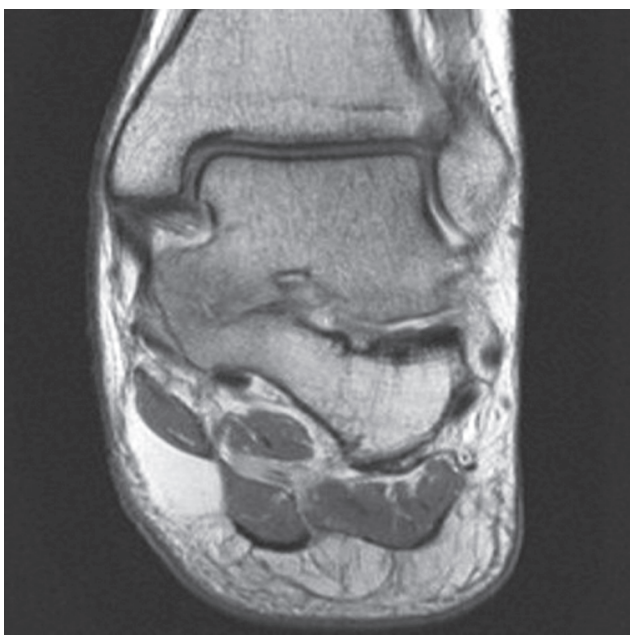

Fig. 4: MRI revealed the type and extent of talocalcaneal coalition

bearing lateral radiograph is required for the surgical plan (Fig. 3A). The first TMT plantar gapping, the sagging and degenerative changes at the NC joint should be considered. The CT and MRI are useful tools to assess for arthrosis as well (Fig. 3B).

Either the medial column arthrodesis or the corrective osteotomy is debatable in terms of the best procedure. The advocates of arthrodesis quote the reliable fusion rates, superb deformity correction, and the insignificant function of the NC and TMT joints. ${ }^{17,24-27}$ The proponents for the Cotton osteotomy dispute that the procedure predictably reconstructs the Meary's line and precludes fusion. ${ }^{28,29}$ The attention should be turned to using the medial column fusion to recognize sagging at the NC joint in the rigid medial column deformity or arthrosis at the NC joint.

Recently, the combined subtalar and NC arthrodesis may be valuable while managing the flatfoot deformity with the NC joint collapse with regard to decrease the stress to the midfoot joints and allow the foot to adjust to the bumpy ground in the coronal plane. ${ }^{30}$

\section{Unrecognized Rigid Deformity}

The unrecognized rigid deformity may lead to reconstruction failure due to the residual pain and the difficulty or inability to achieve good alignment during the corrective osteotomy or the soft tissue procedure. Although most patients with tarsal coalition present between the ages of 8 and 16 years, the symptomatic coalitions occur in the adult population (Fig. 4). This entity should be suspected in the young adult with the rigid painful flatfoot and

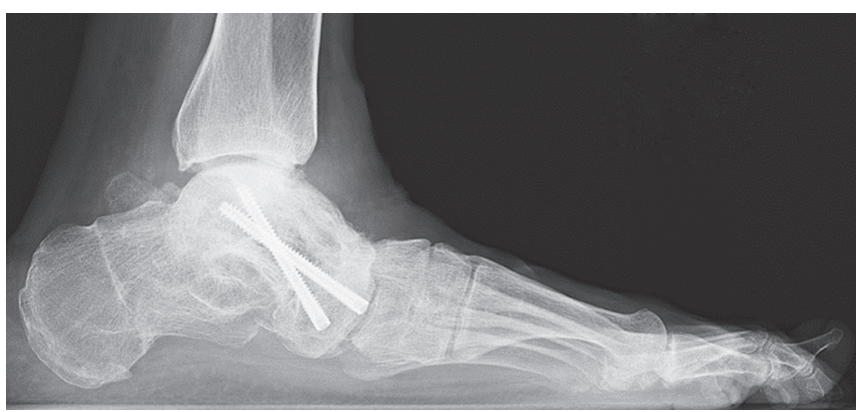

Fig. 5: Rigid flatfoot deformity in a 66-year-old woman with rigid flatfoot deformity had undergone corrective osteotomy with triple arthrodesis which was followed by sagging at the naviculocuneiform joint from adjacent joint arthrosis

lack of motion in the subtalar joint in addition to possible peritalar subluxation. ${ }^{31}$ The CT scan is helpful to identify the osseous coalition. Magnetic resonance imaging can be applied to visualize the fibrocartilaginous coalition.

There is limited literature on simultaneous surgical correction of the flatfoot deformity and the coalition resection. The study showed the combined procedures provided a good result. ${ }^{32}$ It is important to remember that mild flatfoot deformity is likely to be an asymptomatic flatfoot deformity and the coalition resection alone is reasonable. Albeit, the severe flatfoot condition is commonly symptomatic from the flatfoot deformity itself. It is still probably best to correct the deformity in the patient with moderate-tosevere flatfoot deformity. If the coalition is asymptomatic and the restriction of motion does not interfere with gaining good alignment, the coalition can be left alone. The coalition resection should be considered if this procedure will be beneficial to achieve better alignment. The subtalar arthrodesis is more likely to be necessary in the case of an extensive talocalcaneal coalition.

\section{Complications Related to Arthrodesis}

The success rates for the triple arthrodesis generally vary above $85 \%$ all through the article in the rigid flatfoot with arthrosis. ${ }^{33}$ However, nonunion is the most frequent problem reported after the triple arthrodesis. Even though the incidence of nonunion has declined from the improvement of implant design and utilization of biological enhancement, the rates of recurrent deformity and adjacent joint arthrosis have not changed significantly and remain the cause of failure $^{34}$ (Fig. 5). (For more information on arthrodesis, see Arthithat Kirinpanu's article, "Role of arthrodesis in $A A F^{\prime \prime}$ in this issue.) 


\section{Failure to Address the Deltoid Insufficiencies}

The soft tissue components are involved and eventually will bring in the talar tilt with consequent valgus ankle joint from the deformity progression. It is crucial to recognize the valgus ankle component during clinical evaluation. Posterior tibial tendon dysfunction stage IV A (flexible valgus ankle) can be carried out with the selective hindfoot arthrodesis based upon the pathology but the main procedure for the ankle realignment is the deltoid ligament complex restoration. (For more information on PTTD stage IV management, see Thanarat Hempongpun and Chamnanni Rungprai's article, "Stage 4 AAFD: management options" in this issue.)

\section{SUMMARY}

Failed flatfoot reconstruction is a complicated condition that is challenging for the management. The comprehensive history taking and the physical examination, including a thorough assessment of which procedure was achieved throughout the prior surgery, are essential to realize this complex problem. The organized approach to formulating procedures that improve the remaining or recurring deformity is crucial to accomplishing the acceptable correction and patient fulfillment. The simple osteotomies may be sufficient. Alternatively, the arthrodesis may be demanded to obtain the plantigrade foot. The combined soft tissue procedures, such as, tendon transfer or lengthening, may be required as the adjunctive surgery.

\section{References}

1. Bluman EM, Title $\mathrm{Cl}$, Myerson MS. Posterior tibial tendon rupture: a refined classification system. Foot Ankle Clin 2007;12(2):233-249. DOI: 10.1016/j.fcl.2007.03.003.

2. Funk DA, Cass JR, Johnson KA. Acquired adult flat foot secondary to posterior tibial-tendon pathology. J Bone Joint Surg Am 1986;68(1):95-102. DOI: 10.2106/00004623-198668010-00012.

3. Vulcano E, Deland JT, Ellis SJ. Approach and treatment of the adult acquired flatfoot deformity. Curr Rev Musculoskelet Med 2013;6(4):294-303. DOI: 10.1007/s12178-013-9173-z.

4. Jahss MH. Tendon disorders of the foot and ankle. In: Jahss MH, ed. Disorders of the Foot and Ankle. Philadelphia:W.B.Saunders Co; 1991. pp. 1484-1509.

5. Saltzman CL, El-Khoury GY. The hindfoot alignment view. Foot Ankle Int 1995;16(9):572-576. DOI: 10.1177/107110079501600911.

6. Malicky ES, Crary JL, Houghton MJ, et al. Talocalcaneal and subfibular impingement in symptomatic flatfoot in adults. J Bone Joint Surg Am 2002;84(11):2005-2009. DOI: 10.2106/00004623-200211000-00015.

7. Jeng CL, Rutherford T, Hull MG, et al. Assessment of bony subfibular impingement in flatfoot patients using weight-bearing CT scans. Foot Ankle Int 2019;40(2):152-158. DOI: 10.1177/1071100718804510.

8. Johnson KA, Strom DE. Tibialis posterior tendon dysfunction. Clin orthopaedics Relat Res 1989;239(239):196-206. DOI: 10.1097/00003086-198902000-00022.

9. Hunt KJ, Farmer RP. The undercorrected flatfoot reconstruction. Foot Ankle Clin 2017;22(3):613-624. DOI: 10.1016/j.fcl.2017.04.003.

10. Myerson MS, Badekas A, Schon LC. Treatment of stage II posterior tibial tendon rupture with flexor digitorum longus tendon transfer and calcaneal osteotomy. Foot Ankle Int 2004;25(7):445-450. DOI: $10.1177 / 107110070402500701$.

11. Guyton GP, Jeng C, Krieger LE, et al. Flexor digitorum longus transfer and medial displacement calcaneal osteotomy for posterior tibial tendon dysfunction: a middle-term clinical follow-up. Foot Ankle Int 2004;22(8):627-632. DOI: 10.1177/107110070102200802.
12. Abdulmassih S, Phisitkul P, Femino JE, et al. Triceps surae contracture: implications for foot and ankle surgery. J Am Acad Orthop Surg 2013;21(7):398-407. DOI: 10.5435/00124635-201307000-00005.

13. DiGiovanni CW, Langer P. The role of isolated gastrocnemius and combined achilles contractures in the flatfoot. Foot Ankle Clin 2007;12(2):363-379. DOI: 10.1016/j.fcl.2007.03.005.

14. Arangio GA, Salathé EP. Medial displacement calcaneal osteotomy reduces the excess forces in the medial longitudinal arch of the flat foot. Clin Biomech (Bristol, Avon) 2001;16(6):535-539. DOI: 10.1016/ S0268-0033(01)00011-0.

15. Griend RV. Lateral column lengthening using a " $Z$ " osteotomy of the calcaneus. Techniq Foot Ankle Surg 2008;7(4):257-263. DOI: 10.1097/ BTF.0b013e318183a0df.

16. Deland JT. Adult-acquired flatfoot deformity. J Am Acad Orthop Surg 2008;16(7):399-406. DOI: 10.5435/00124635-200807000-00005.

17. Barg A, Brunner S, Zwicky L, et al. Subtalar and naviculocuneiform fusion for extended breakdown of the medial arch. Foot Ankle Clin 2011;16(1):69-81. DOI: 10.1016/j.fcl.2010.11.004.

18. Chan JY, Greenfield ST, Soukup DS, et al. Contribution of lateral column lengthening to correction of forefoot abduction in stage Ilb adult acquired flatfoot deformity reconstruction. Foot Ankle Int 2015;36(12):1400-1411. DOI: 10.1177/1071100715596607.

19. laquinto JM, Wayne JS. Effects of surgical correction for the treatment of adult acquired flatfoot deformity: a computational investigation. J Orthop Res 2011;29(7):1047-1054. DOI: 10.1002/jor.21379.

20. Tien T, Parks B, Guyton G. Plantar pressures in the forefoot after lateral column lengthening: a cadaver study comparing the evans osteotomy and calcaneocuboid fusion. Foot Ankle Int 2005;26(7):520-525. DOI: $10.1177 / 107110070502600704$.

21. Cooper P, Nowak M, Shaer J. Calcaneocuboid joint pressures with lateral column lengthening (Evans) procedure. Foot Ankle Int 1997;18(4):199-205. DOI: 10.1177/107110079701800403.

22. Henry JK, Shakked R, Ellis SJ. Adult-acquired flatfoot deformity. Foot Ankle Orthop 2019;4(1):1-17. DOI: 10.1177/2473011418820847.

23. Benthien RA, Parks BG, Guyton GP, et al. Lateral column calcaneal lengthening, flexor digitorum longus transfer, and opening wedge medial cuneiform osteotomy for flexible flatfoot: a biomechanical study. Foot Ankle Int 2007;28(1):70-77. DOI: 10.3113/FAI.2007.0013.

24. Greisberg J, Assal M, Hansen ST, et al. Isolated medial column stabilization improves alignment in adult-acquired flatfoot. Clin Orthop Relat Res 2005(435):197-202. DOI: 10.1097/01. blo.0000157658.17056.46.

25. Greisberg J, Hansen Jr ST, Sangeorzan B. Deformity and degeneration in the hindfoot and midfoot joints of the adult acquired flatfoot. Foot Ankle Int 2003;24(7):530-534. DOI: 10.1177/107110070302400704.

26. Ajis A, Geary N. Surgical technique, fusion rates, and planovalgus foot deformity correction with naviculocuneiform fusion. Foot Ankle Int 2014;35(3):232-237. DOI: 10.1177/1071100713517098.

27. JackEA. Naviculo-cuneiform fusion in the treatment of flat foot. J Bone Joint Surg Br 1953;35-B(1):75-82. DOI: 10.1302/0301-620X.35B1.75.

28. Aiyer A, Dall GF, Shub J, et al. Radiographic correction following reconstruction of adult acquired flat foot deformity using the cotton medial cuneiform osteotomy. Foot Ankle Int 2016;37(5):508-513. DOI: $10.1177 / 1071100715620894$.

29. Lutz M, Myerson M. Radiographic analysis of an opening wedge osteotomy of the medial cuneiform. Foot Ankle Int 2011;32(3):278287. DOI: 10.3113/FAI.2011.0278.

30. Steiner CS, Gilgen A, Zwicky L, et al. Combined subtalar and naviculocuneiform fusion for treating adult acquired flatfoot deformity with medial arch collapse at the level of the naviculocuneiform joint. Foot Ankle Int 2019;40(1):42-47. DOI: 10.1177/1071100718800295.

31. Vincent KA. Tarsal coalition and painful flatfoot. J Am Acad Orthop Surg 1998;6(5):274-281. DOI: 10.5435/00124635-199809000-00002.

32. Mosca VS, Bevan WP. Talocalcaneal tarsal coalitions and the calcaneal lengthening osteotomy: the role of deformity correction. J Bone Joint Surg Am 2012;94(17):1584-1594. DOI: 10.2106/JBJS.K.00926. 
33. Coetzee JC, Hansen ST. Surgical management of severe deformity resulting from posterior tibial tendon dysfunction. Foot Ankle Int 2001;22(12):944-949. DOI: 10.1177/1071100701022 01202.
34. Sammarco VJ, Magur EG, Sammarco GJ, et al. Arthrodesis of the subtalar and talonavicular joints for correction of symptomatic hindfoot malalignment. Foot Ankle Int 2006;27(9):661-666. DOI: $10.1177 / 107110070602700901$. 